\title{
MOLECULAR CHARACTERIZATION OF SOME Medicago SPECIES
}

(Received: 22.7.2010)

\author{
By \\ G. Tarabin, G. Al Amir, and S. Lawand* \\ Department of Agronomy, Faculty of Agriculture, Damascus University, Syria \\ *Department of Medicinal and Aromatic Plants, General Commission for Scientific \\ Agricultural Research, Syria
}

\begin{abstract}
This investigation was carried out at the Laboratory of Biotechnology at the Faculty of Agriculture - University of Damascus, during the season 2009-2010. Ten wild species and a cultivated one (as a control) were planted to study the genetic diversity among them and to determine the degree of genetic similarity using the ISSR (Inter Simple Sequence Repeats) technique. The used primers proved their effectiveness in showing polymorphism among the studied species and the control. Primers gave a total of 92 allele with a polymorphic percentage of $100 \%$. The number of bands for each primer varied from a minimum of 5 bands for the primer (ISSR34) to a maximum of 21 bands for the primer (ISSR-41) with an average of 11.5 bands for each primer. Cluster analysis and Dendrogram showed the highest degree of genetic similarity between species $M$. rotata and $M$. polymorpha $(0.23)$, while it was low between species $M$. orbicularis and both species $M$. rotata and $M$. intertexta (0.57). Results showed a vast genetic diversity among the studied species.
\end{abstract}

Key words: genetic diversity, medicago, molecular characterization.

$$
\begin{aligned}
& \text { التوصيف الجزيئي لبعض أنواع الجنس Medicago } \\
& \text { جورج طربين -غيداء الأمير- سلام لاوند* }
\end{aligned}
$$

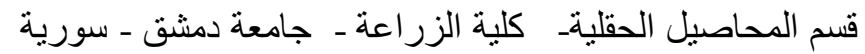

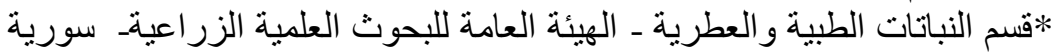

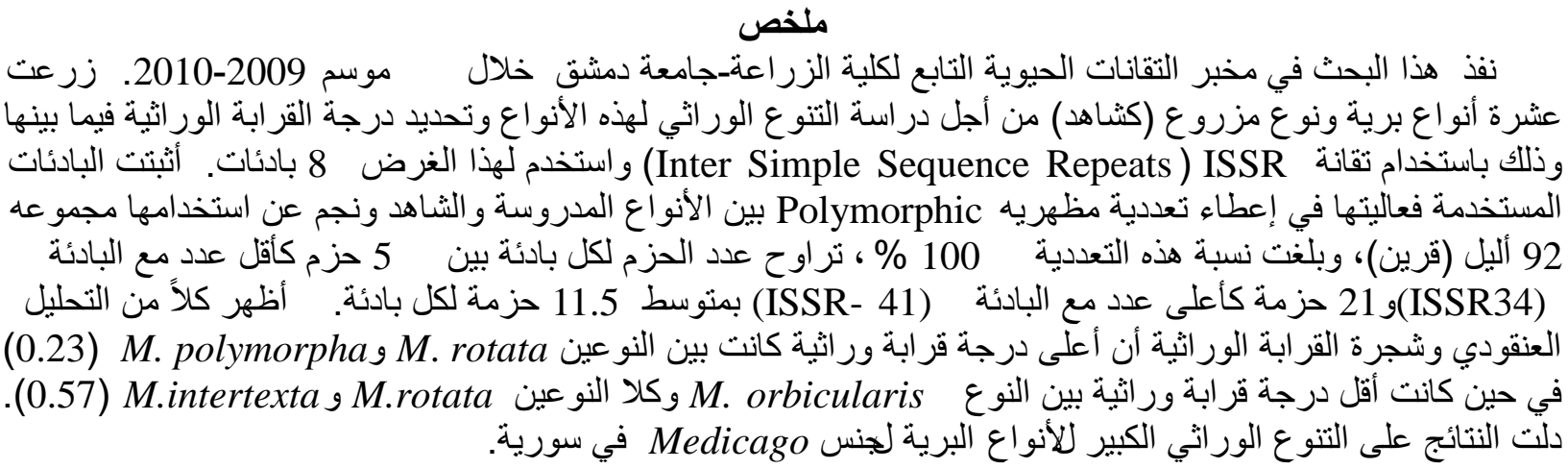


ورغم أهمية الصفات الثكلية والخصائص الف سيولوجية

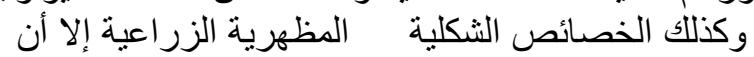
الحاجة للمؤشرات الجزيئية أصبحت أكثر أهمية و إلحاحاً ويرجع ذللك للأسباب التالية:

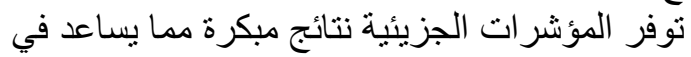

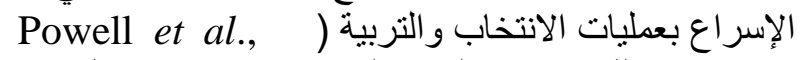
1996)، وبذلك تختصر الزمن الذي تستغرقه إضافة إلى الى التى خفض كلفة المادة التي تحتاجها الدراسات النات

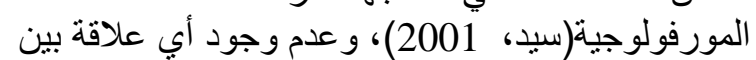

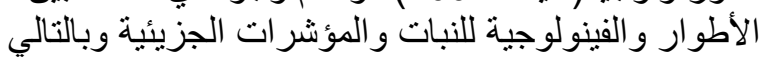

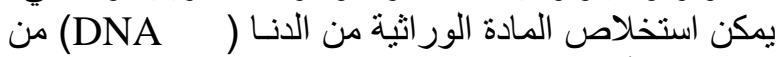

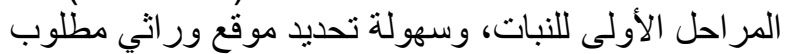

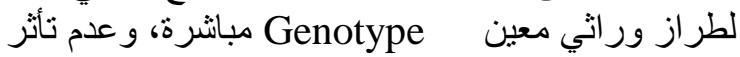
المؤشر ات بالثكل الظاهري للنباتات و المؤشر ات البيئية كما

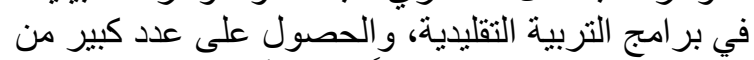

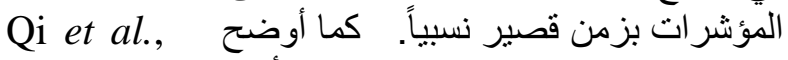
1996, Ramsay et al., 2000

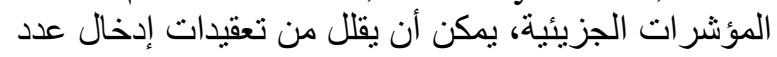

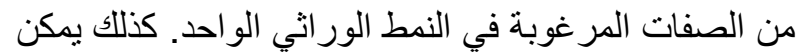

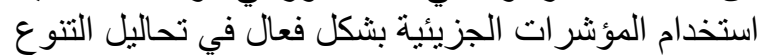
Powell et al., 1996; ) الوراثي وتقدير التشابه الور التئية .Eleuch et al., 2008 طُور التفاعل التسلسلي البوليمبرازي (البيسرة) من قبل (Polymerase Chain Reaction- PCR) الباحث (Saiki et al.,1985) الذي كان لله أثراً مهماً على مئى

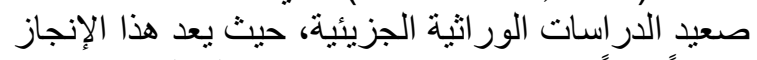

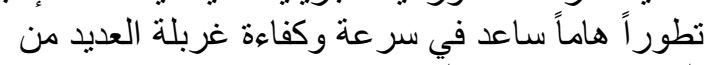

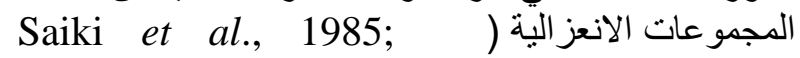
بواعفة (Tragoonrung et al., 1992 بمضاعفة Amplification قطع محددة

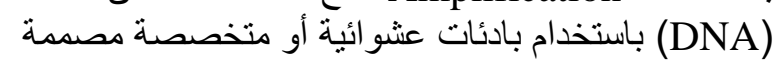

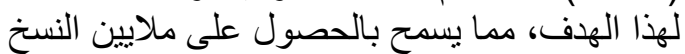

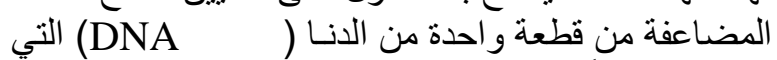
تتضاعف أسياً، وذلك باستخدام دور ات مدات حرارية متعددة سيد، Karp et al., 1997; Ayad et al., 1997) 2001). وقد ساعد تصنيع أجهزة التدوير الحراري التهاي automated thermo cycler

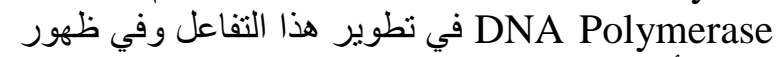

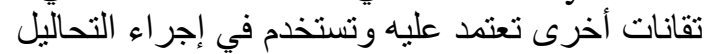

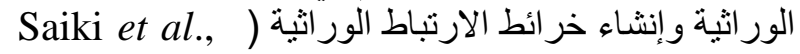

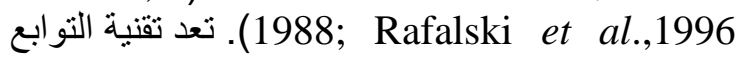
التر ادفية البسيطة الداخلية ( Inter Simple Sequence

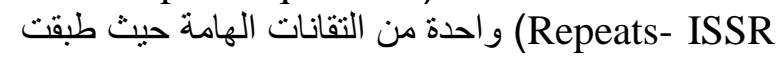

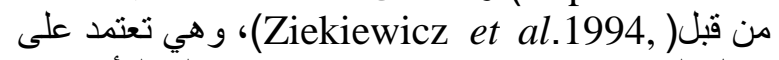
فاعل البيسرة (PCR) ومؤشر ات جزيئية مثالية للأسبـاب

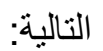

\section{1}

تمناز سورية بتباينات جغر افية ومناخية وبيئية لها

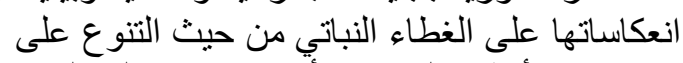

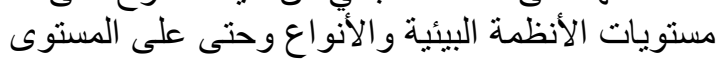

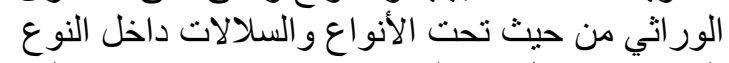

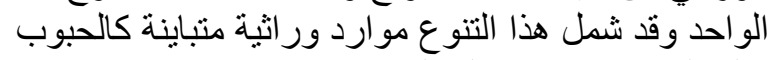

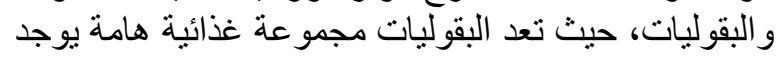

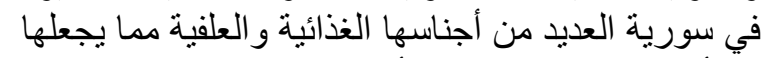

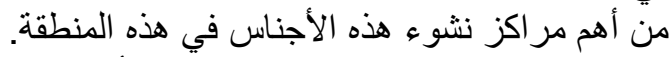

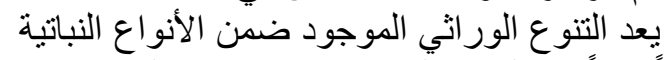

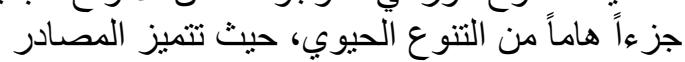

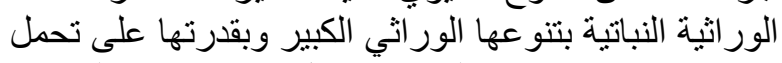

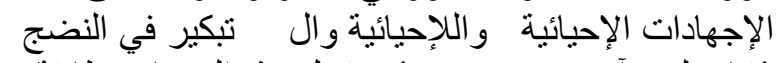

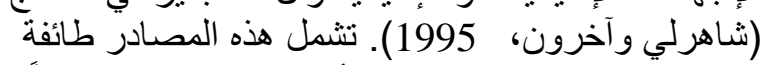

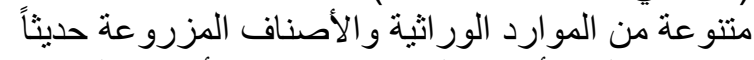

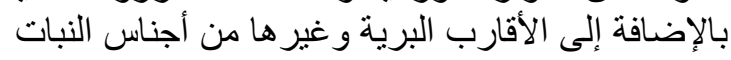

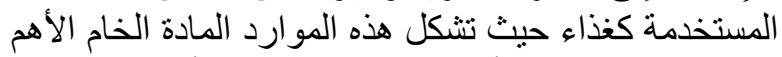

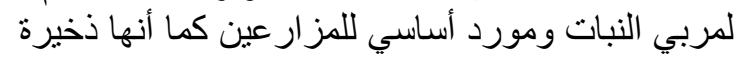

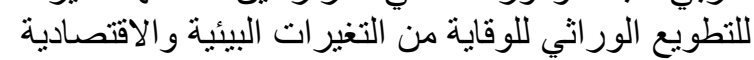

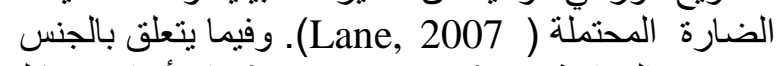

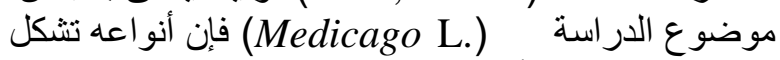

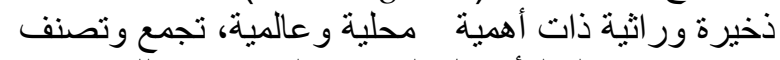

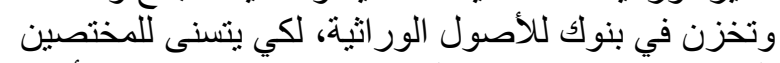

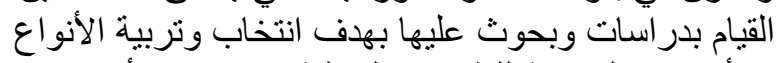

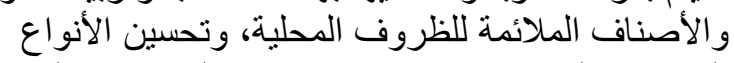

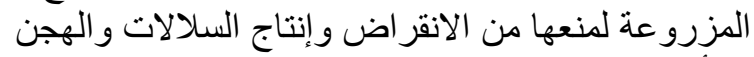

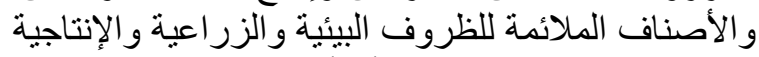

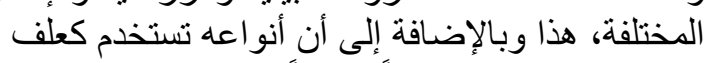

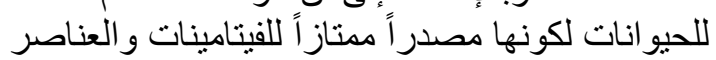

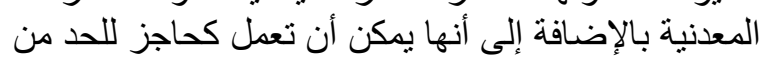

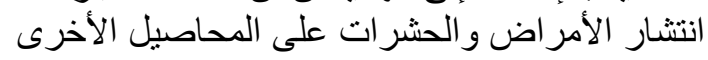

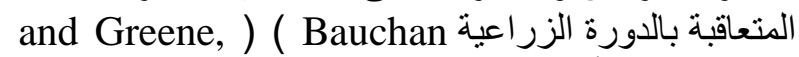

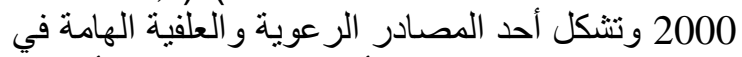

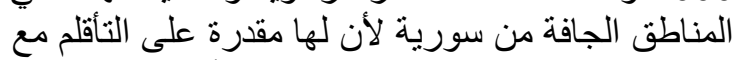

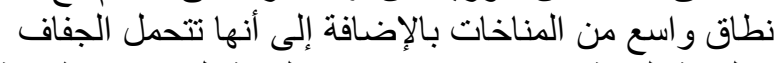

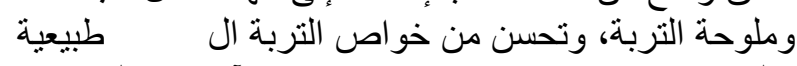

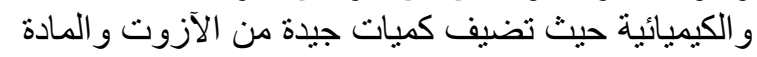

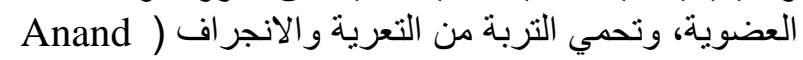
. et al., 2000

تشكل محاو لات البحث عن معايير جديدة للتمبيز بين

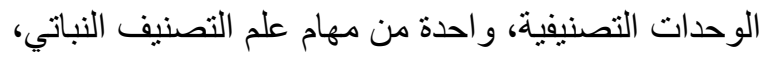

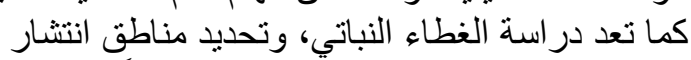

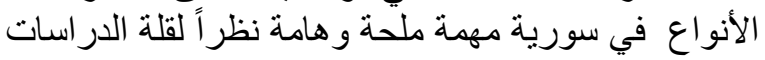

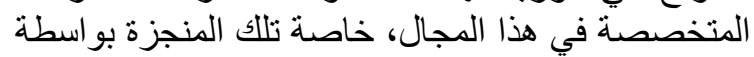

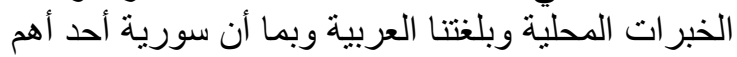

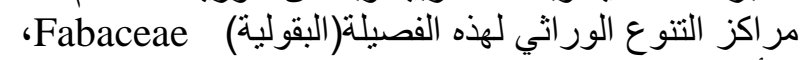

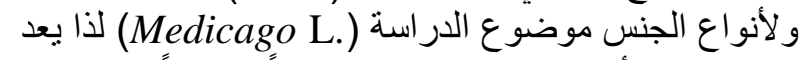
حصر هذه الأنو اعو ونوصنيفها بدقة ور اثياً (جزيئياً)

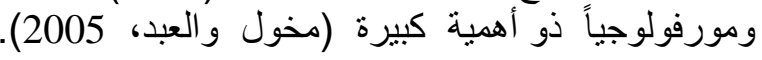




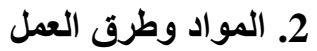

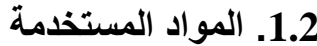

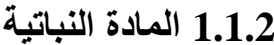

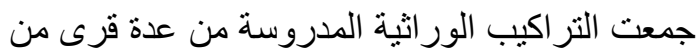

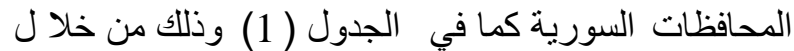
القيام بالعديد من جو لات الجمع الحقلية.

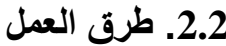

1.2.2 تعقيم البذور وزراعتها

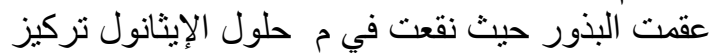

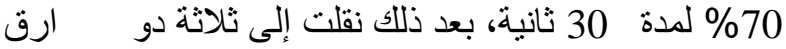

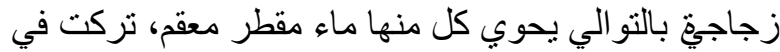

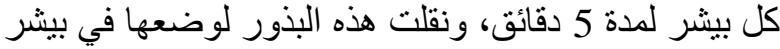

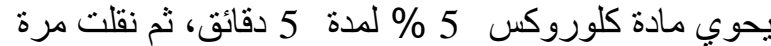

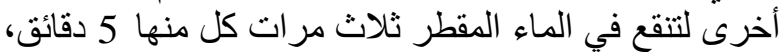

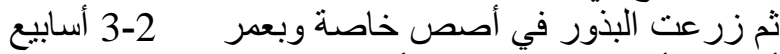

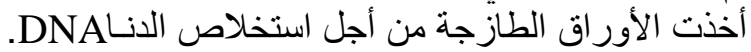

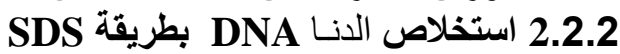

طدن 1جرام من الأوراق الخضر اءع باء باستخدام الآزوت

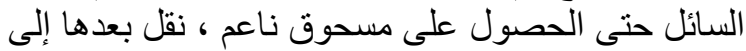

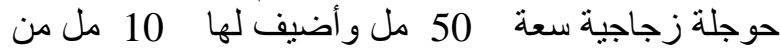

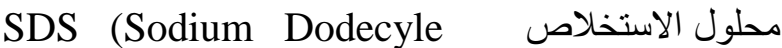
(0.1 M Tris-HCl,PH=8.2, و المكون من Sulphate) 50mM EDTA, o.1M NaCl, 2\% SDS, $1 \mathrm{mg} / \mathrm{ml}$

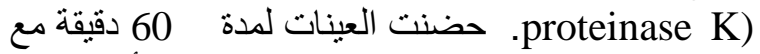

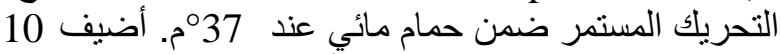

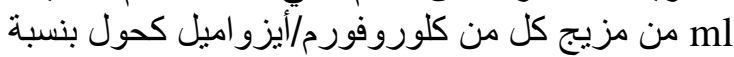

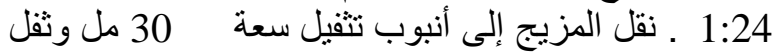

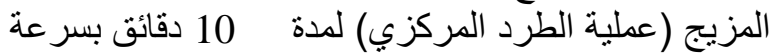

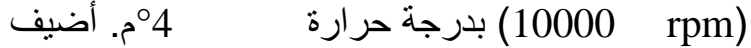

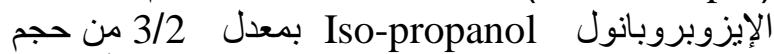

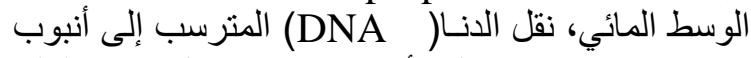

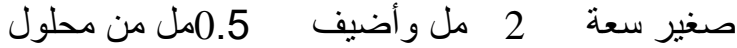

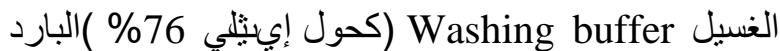
(المحفوظ بدرجة - 20 مُ) بالتثفيل بسر عة ( 10000 rpm)

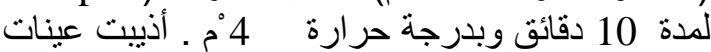

الدنـا(DNA) في 500 ميكروليتر من المحلول المنظم

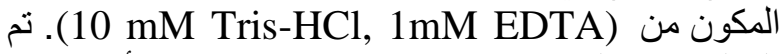

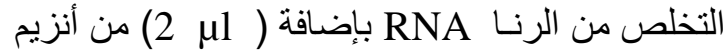

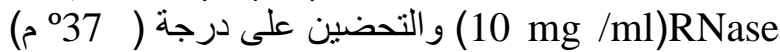

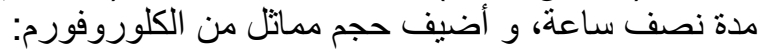
ايزوميل الكحول (1:24). وبعد التثفيل ونقل الطور العلوي الطوي

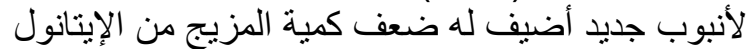

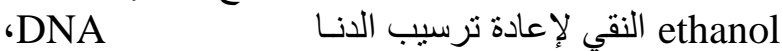

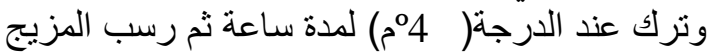
بالتثفيل بسر عة (10000rpm) و لمدة 10 دقائق و وغسل ثنانية بواسطة الإبيثنول 70\% و جفف في الهو اء للتخلص
تُضخم منطقة التو ابع التر ادفية البسيطة ويستخدم بادئ

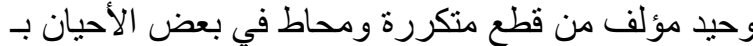

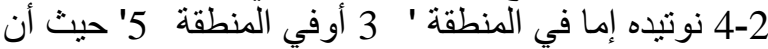

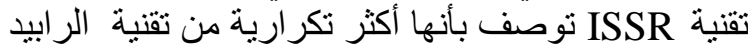

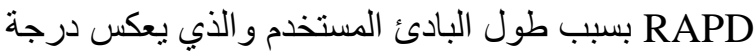

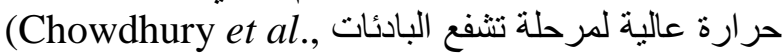

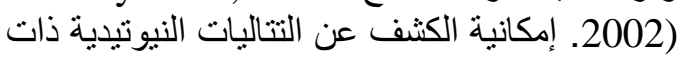

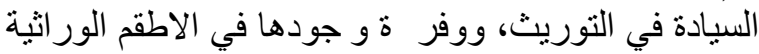

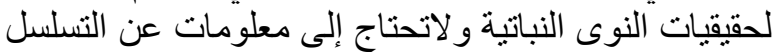

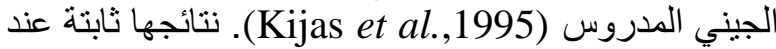
تكر ارها و وسريعة كما أنها تتطلب كمية قليلة من

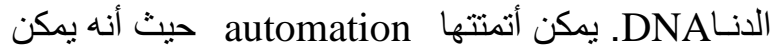

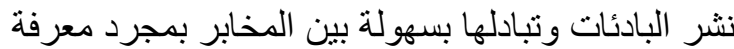

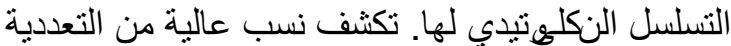

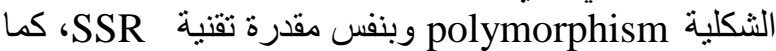
استخدمت هذه التقنية لدر اسة التنوع الور اثي في

(Bornet et al., 2002) والبطعير (Ferández et)

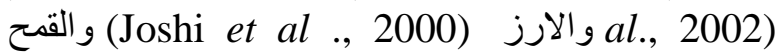
.(Nagaoka and Ogihara, 1997) قام Petolescu and Nedelea (2009) بدر اسة

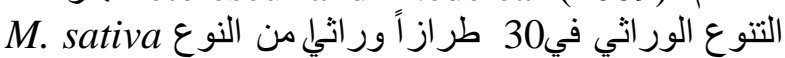

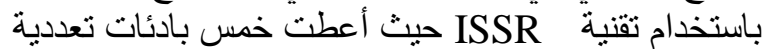

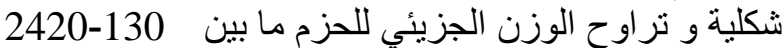

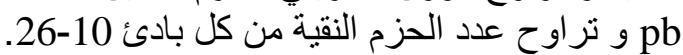
قام Touil et al., (2008) بدر اسة 26 عشيرة نباتية

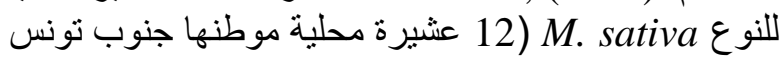

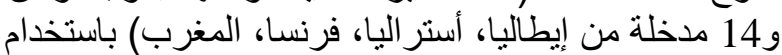

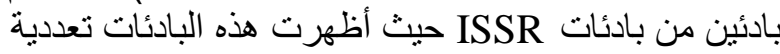

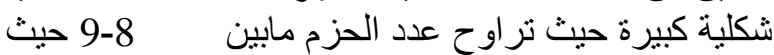
انقسمت هذه العشائر إلى 4 مجمو عات كبرد كبيرة نم الحصول

أجرى et al., Valizadeh عليها. أنواع تعود إلى أربعة تحت أجناس تنتمي للجنس Medicago 132 من حزم الريفليبات RFLP والتي تم التمات الحصول عليها

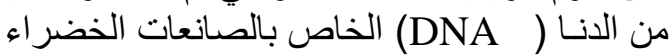

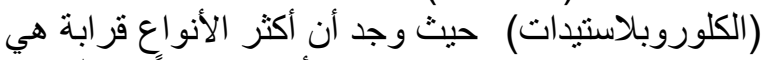
 .M.lupulina

أجرى et al., Sigovia-Lerma

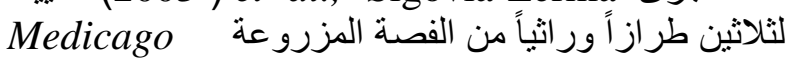
34 sativa L.

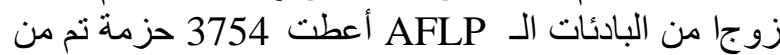
خلالها التعرف على الباني 1541 حزمة ذات الت اتعدات تعدية شكلية. كان

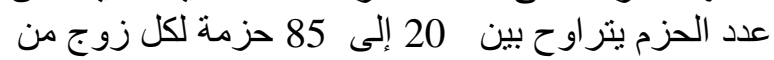
البادئات 


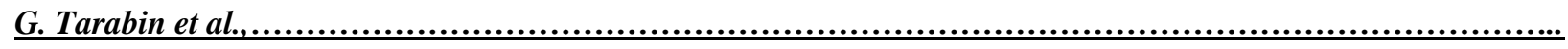

\begin{tabular}{|c|c|c|c|c|c|c|}
\hline \multirow{2}{*}{ خط العرض N } & \multirow{2}{*}{$\begin{array}{c}\text { خط الطول } \\
\text { E }\end{array}$} & \multirow{2}{*}{ الارتفاع } & \multicolumn{2}{|c|}{ منطقة الجمع } & \multirow{2}{*}{ الاسم العلمي للانواع المدروسة } & \multirow{2}{*}{ الددروسة } \\
\hline & & & 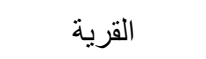 & المحافظة & & \\
\hline 32.595 & 36.58 & 430 & قلعة صلاح الدين & التلاذقية & M. minima Lam. & نوع بري \\
\hline 33.717 & 36.107 & 1110 & 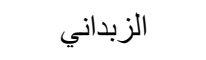 & د مشق & M. rigidula (L.) Desr. & نوع بري \\
\hline 34.709 & 36.613 & 200 & 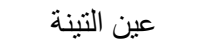 & حمص حص & M. rotata Boiss. & 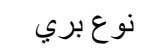 \\
\hline 34.673 & 36.232 & 300 & 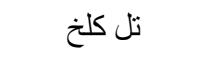 & 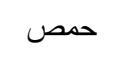 & M. intertexta (L.)Miller & نوع بري \\
\hline 36.215 & 36.597 & 380 & 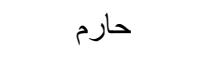 & ادلب & M. blancheana Boiss. & نوع بري \\
\hline 35.758 & 36.363 & 230 & جسر الشغور & 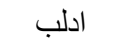 & M. polymorpha $\mathrm{L}$. & ن بري \\
\hline 35.918 & 35.973 & 800 & كسب & اللاذقية & M. turbinata (L.) Willd. & ن بري \\
\hline 35.088 & 36.197 & 970 & 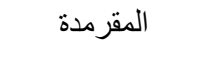 & 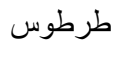 & M. scutellata Mill. & نوع بري \\
\hline 33.717 & 36.107 & 1110 & 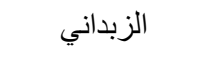 & 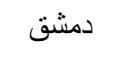 & M. orbicularis (L.) All. & نوع بري \\
\hline 33.682 & 36.100 & 1300 & الزبداني & 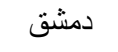 & M. sativa $\mathrm{L}$. & نوع مزروع \\
\hline 35.148 & 36.092 & 740 & الحطانية & طرطوس & M. truncatula Gaerth. & ن بري \\
\hline
\end{tabular}

جدول رقم (2) : التسلسل النكليوتيدي للبادئات المختبرة في تقاتة النيلة النكليوني

\begin{tabular}{|l|c|}
\hline \multicolumn{1}{|c|}{ '5 - التسلسل النوتيدي 1 ' البائة } & \\
\hline AGAGAGAGAGAGAGAGC & ISSR-32 \\
\hline GAGAGAGAGAGAGAGAT & ISSR-33 \\
\hline CTCTCTCTCTCTCTCTG & ISSR-34 \\
\hline CACACACACACAACAG & ISSR-35 \\
\hline TCTCTCTCTCTCTCTCC & ISSR-36 \\
\hline TGTGTGTGTGTGTGTGG & ISSR-37 \\
\hline ACACACACACACACACTT & ISSR-40 \\
\hline ACACACACACACACACGG & ISSR-41 \\
\hline
\end{tabular}

(Fermentas, التفاعل الذي نم الحصول عليه من شركة) :Germany) ويتم هذا التفاعل في جهاز التدوير الحراري وفقاً للظروف

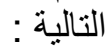

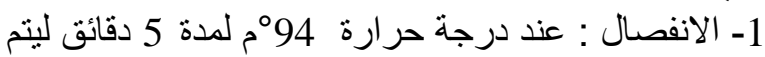
انفصال سلسلتي الدنـا.

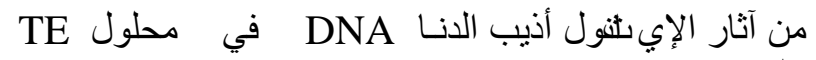

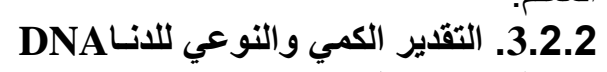

استخدم جهاز

Instruments, Inc.) نقاوته حيث بعتمد الجهاز في عمله على قياس كمية لإنا

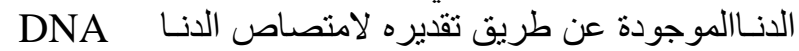
للأشعة فوق البنفسجية بموجات طولهائل

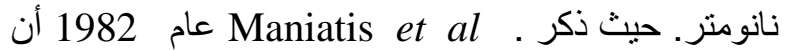
النسبة بين قراءة الموجة 260 نائر 260 نانو متر و الموجة 280

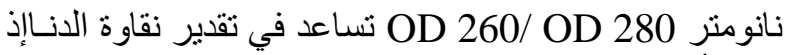

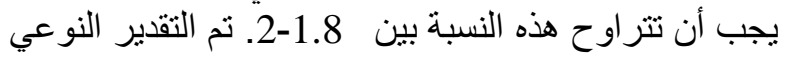

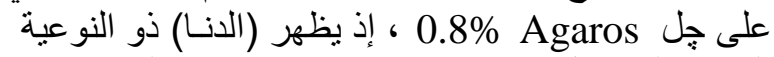

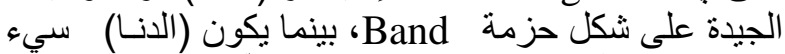

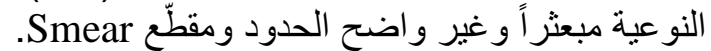

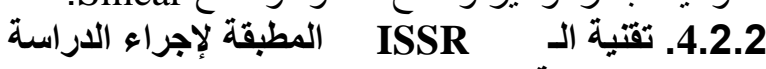
الجزيئية

اختبر(8) بادئات تم الحصول عليها من الهيئة العامة

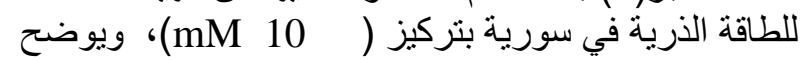
الجدول رقم ( 2) التسلسل النوتيدي للبادئات المستخدمة في

أجري تفاعل البيسره PCR وفقاً لطريقة

et al., 1990 النهائي (25 ) كما يظهر الجدول رقم (3) مكونات هذان 
Image الناتجة عن التضخيم. تم تصوير الجهل Agle Eye II ) Analyzer باستخدام جهاز .(Staratagene 3.2. تحليل النتائج

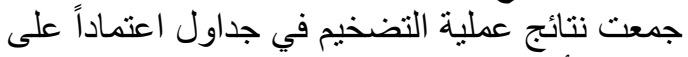

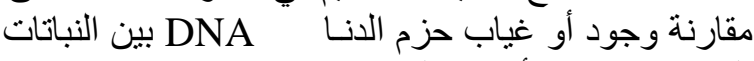

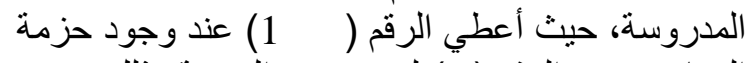

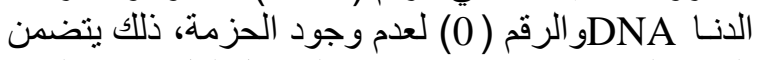

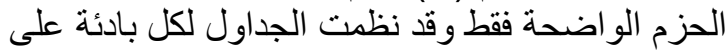
Dدى، ورسمت شجرة القر ابة الور اثية بتطبيق منوسطات المجمو عات الزوجية غير الرزاتية UPGMA Unweighted Pair Group المزية المغئن Method with Arithmetic Averaging Nei الإحصائي طبقا لطريقة 1.31 Pop gene .(1987).

\section{3.النتائج والمناقشة}

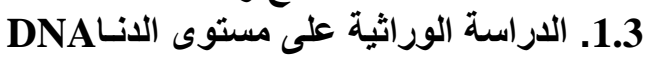

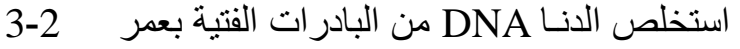

أسابيع وقيس تركيزه ونقاوته بجهاز المطياف الضونئي حيث التئي

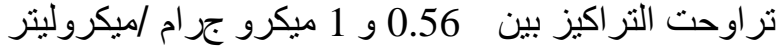

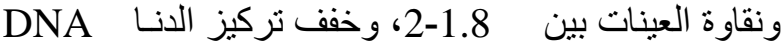

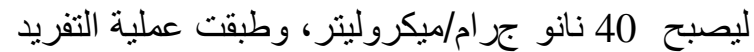

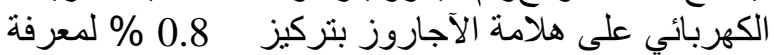

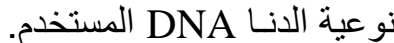
ثم طبقت تقنية ISSR باستخدام 8 بادئات ـ فأعطت البادئات الثماني حزم و اضحة وذات تعددية شكلية كما يبين بانئ

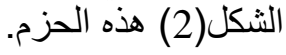

جدول رقم (3) : مكونات تفاعل البلمرة المتسلسل PCR

\begin{tabular}{|c|c|}
\hline مكونات تفاعل البيسره PCR & الكميات \\
\hline Taq DNA Polymerase & 0.05 units $/ \mu \mathrm{l}$ \\
\hline MgCl2 & $4 \mathrm{Mm}$ \\
\hline dNTPs & $2.5 \mu \mathrm{l}$ \\
\hline DNA & $2 \mu \mathrm{l}(25 \mathrm{ng} / \mu \mathrm{l})$ \\
\hline Primer & $2.5 \mu \mathrm{l}(10 \mathrm{pmol} / \mu \mathrm{l})$ \\
\hline Buffer & $10 X$ \\
\hline $\mathrm{H}_{2} \mathrm{O}$ & \\
\hline
\end{tabular}

2- 40 دورة تتضمن كل منها المر احل التالية:التحطم :

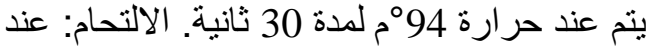

حر ارة 51 م لمدة دقبقة واحدة ـ الاستطالة: عند حرارة



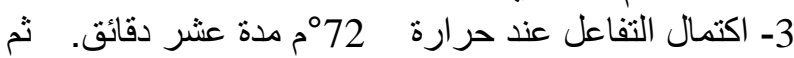

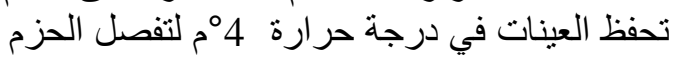

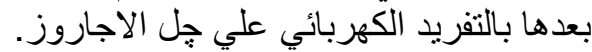

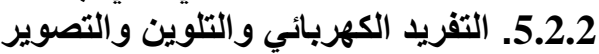

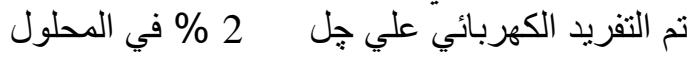
TBE 1X المنظم المر

$(10 \mathrm{X}$ TBE buffer $=108 \mathrm{~g}$ Tris borate $+55 \mathrm{~g}\}$ و و المضاف اليها (10 mg/ml من صبغة الايثيديوم برومايد

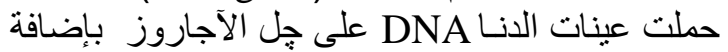

5 ميكرولتر من سائل التحميل الخاص ( ) 1X Loading (buffer Bromophenol blue و المكنون من (15\%) Ficoll $400+1.03 \%$ bromophenol Blue +0.03 $\%$ xylene cyanol FF $+0.4 \%$ Orange $\mathrm{G}+10$ $\mathrm{mM}$ Tris- $\mathrm{HCl}+50 \mathrm{mM}$ EDTA)
12
3
4
$5 \quad 6$
67
8
$9 \quad 10$
11

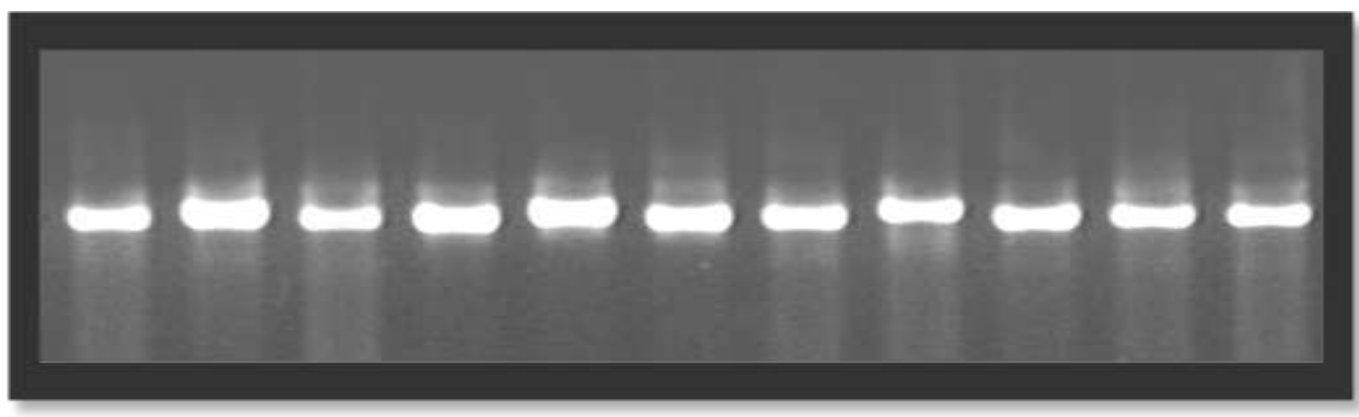

الشكل(1): صورة هلامة الآجاروز بتركيز 0.8\% لتحديد نوعية الدـاDNA. 
1

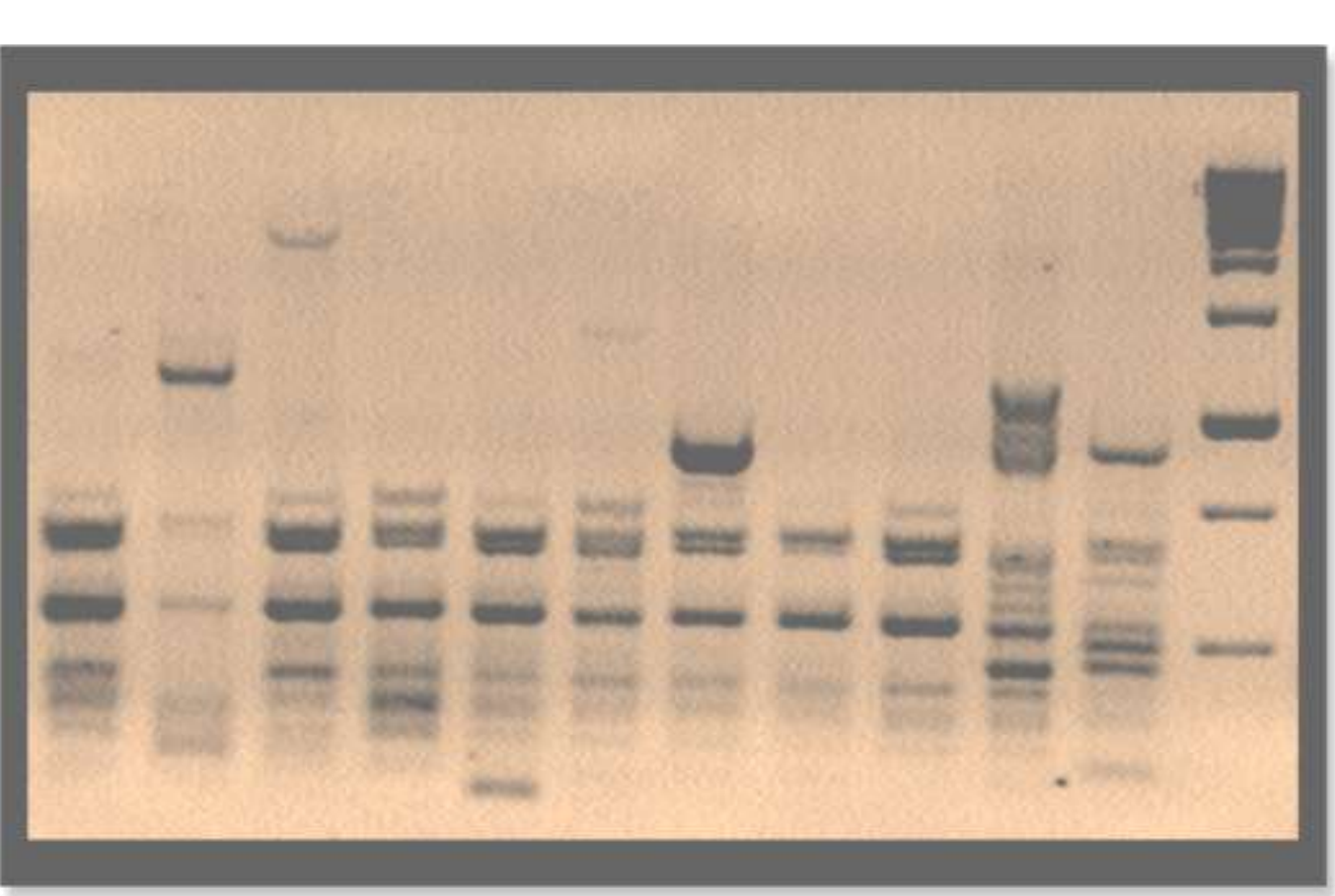

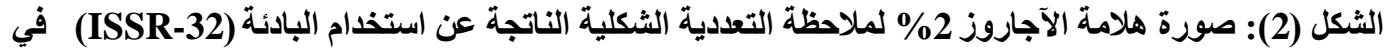

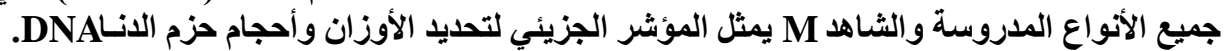

(1): M.minima, (2): M.rigidula, (3): M.rotata, (4): M.intertexta,

(5): M.blancheana, (6): M.polymorpha, (7): M.turbinata, (8): M.scutellata,

(9): M.orbicularis, (10): M.sativa, (11): M.truncatula, M :(1Kb) معلم

وM.intertexta مما يدل على وجود تباين ور اثي كبير

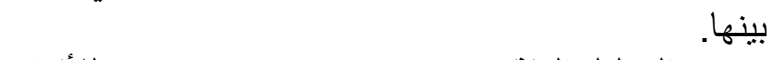

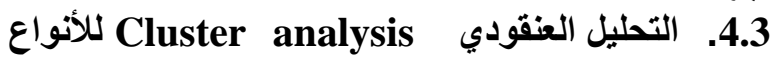

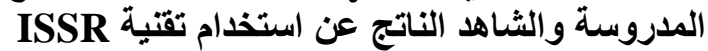

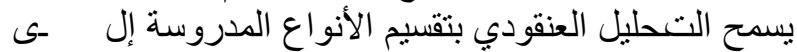

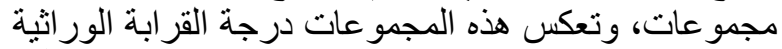

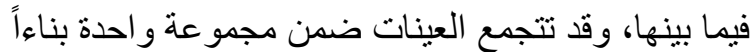

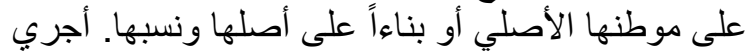

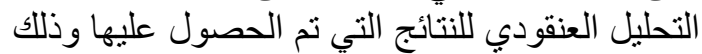

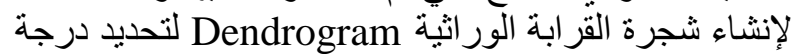

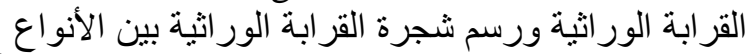

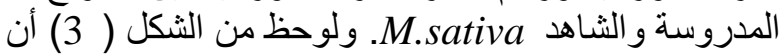

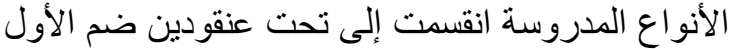

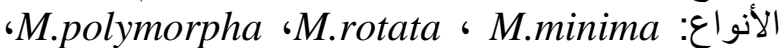
M.intertexta ،M.blancheana M.polymorpha ،M.rotata القرابة الور اثية بمسافة 11.57، في حين ضم تحت العنقود الثاني الأنواع: M.rigidula ‘M.truncatula ‘M.sativa ،M.scutellata M.orbicularis

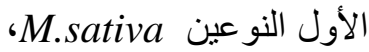

جميع البادئات المستخدمة أعطت منتجات تضخيم في البادي

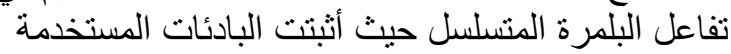

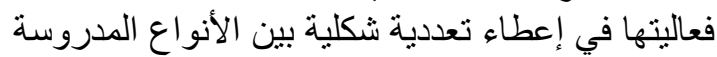

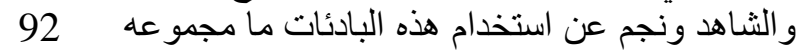

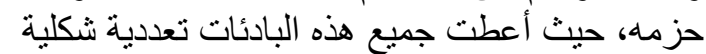
polymorphic

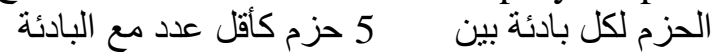
(ISSR-41) بمتوسط 11.5 حزمة لكل بادئة.

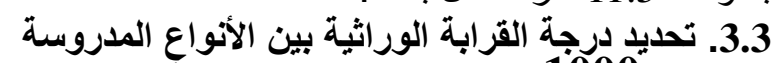

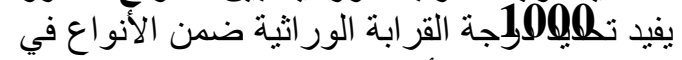

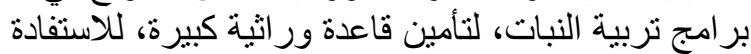

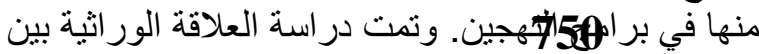

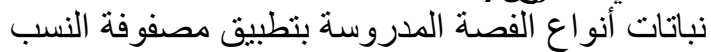

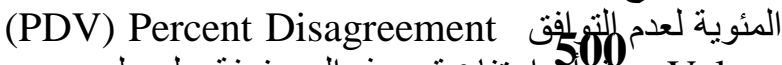
حيlues اختلاف ور اثي وبازديادها يزداد التباين الور اثي بين النباتين باتين

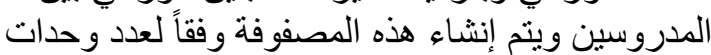

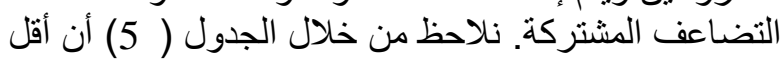

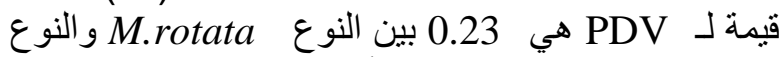
M.polymorpha النوع M.orbicularis و النو عين M.rotata، 
18.41

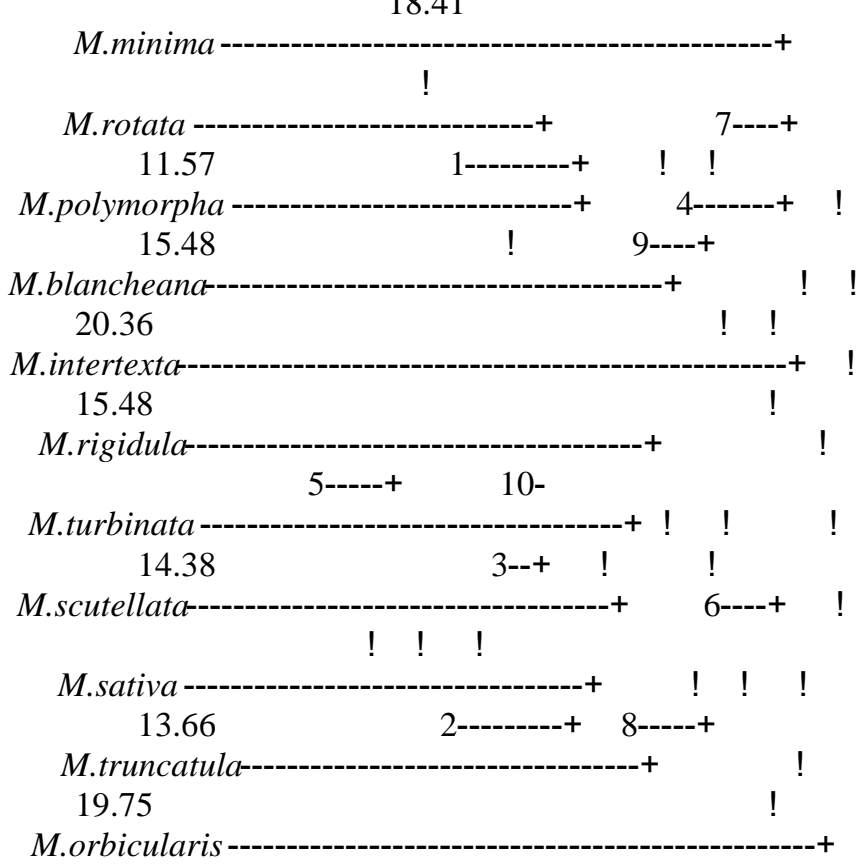

الشكل (3) التحليل العنقودي للأنواع المدروسة والثاهد، الناتج عن استخدام تقنية Cluster Analysis.

\begin{tabular}{|c|c|c|c|}
\hline & \multicolumn{3}{|c|}{ الثكلية \% في الأنواع المدروسة. } \\
\hline النسبة المئوية للتعددية الشكلية\% & عدد الحزم المنباينة & عدد الحزم الكلية & اسم البادئ \\
\hline 100 & 18 & 18 & ISSR-32 \\
\hline 100 & 17 & 17 & ISSR-33 \\
\hline 100 & 5 & 5 & ISSR-34 \\
\hline 100 & 7 & 7 & ISSR-35 \\
\hline 100 & 6 & 6 & ISSR-36 \\
\hline 100 & 7 & 7 & ISSR-37 \\
\hline 100 & 11 & 11 & ISSR-40 \\
\hline 100 & 21 & 21 & ISSR-41 \\
\hline 100 & 92 & 92 & المجموع \\
\hline 100 & 11.5 & 11.5 & المتوسط \\
\hline
\end{tabular}

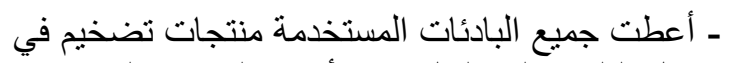

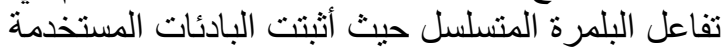

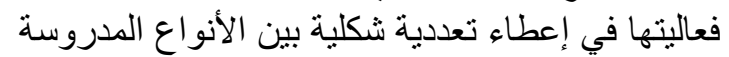

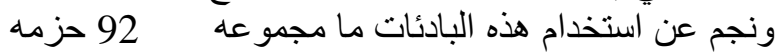

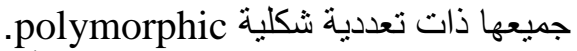
ـ تكثيف الجولات الحقلية لتشمل الجزء أعة الأكبر من مناطق

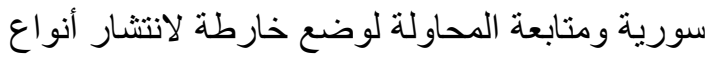
الفصة البرية في سورية.
M.truncatula

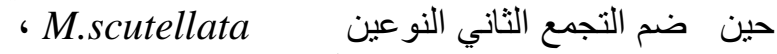
M.turbinata بمسافة ور اثية بلغت 14.38 التين

4. الاستتتاجات والمقترحات

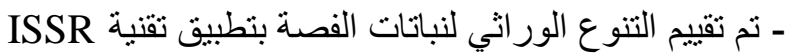

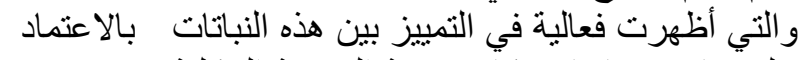

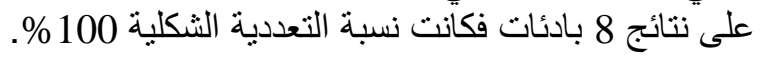


جدول (5) : مصفوفة النسب المئوية لعدم التوافق (PDV) بين الأنواع المدروسة والثاهد والناتجة عن تطبيق متوسطات المجموعات الزوجية غير

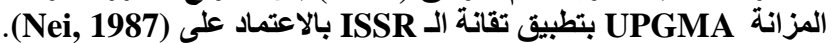

\begin{tabular}{|c|c|c|c|c|c|c|c|c|c|c|c|}
\hline & $\mathbf{1}$ & $\mathbf{2}$ & $\mathbf{3}$ & $\mathbf{4}$ & $\mathbf{5}$ & $\mathbf{6}$ & $\mathbf{7}$ & $\mathbf{8}$ & $\mathbf{9}$ & $\mathbf{1 0}$ & $\mathbf{1 1}$ \\
\hline $\mathbf{1}$ & 0 & & & & & & & & & & \\
\hline $\mathbf{2}$ & 0.41 & 0 & & & & & & & & & \\
\hline $\mathbf{3}$ & 0.36 & 0.51 & 0 & & & & & & & & \\
\hline $\mathbf{4}$ & 0.39 & 0.41 & 0.39 & 0 & & & & & & & \\
\hline $\mathbf{5}$ & 0.36 & 0.44 & 0.30 & 0.46 & 0 & & & & & & \\
\hline $\mathbf{6}$ & 0.38 & 0.39 & 0.23 & 0.38 & 0.32 & 0 & & & & & \\
\hline $\mathbf{7}$ & 0.38 & 0.30 & 0.44 & 0.41 & 0.35 & 0.33 & 0 & & & & \\
\hline $\mathbf{8}$ & 0.33 & 0.32 & 0.43 & 0.43 & 0.39 & 0.41 & 0.29 & 0 & & & \\
\hline $\mathbf{9}$ & 0.39 & 0.44 & 0.57 & 0.57 & 0.43 & 0.48 & 0.38 & 0.36 & 0 & & \\
\hline $\mathbf{1 0}$ & 0.43 & 0.32 & 0.50 & 0.46 & 0.46 & 0.44 & 0.38 & 0.30 & 0.39 & 0 & \\
\hline $\mathbf{1 1}$ & 0.46 & 0.38 & 0.50 & 0.50 & 0.50 & 0.55 & 0.41 & 0.33 & 0.39 & 0.27 & 0 \\
\hline
\end{tabular}

(1): M.minima,(2): M.rigidula, (3): M.rotata,(4): M.intertexta,(5): M.blancheana,(6): M.polymorpha,

(7): M.turbinata, (8): M.scutellata, (9): M.orbicularis, (10): M.sativa, (11): M.truncatula

Bornet B., Goraguer F., Joly G. and Branchard M. (2002). Genetic diversity in European and Argentinean cultivated potatoes (Solanum tuberosum subsp. Tuberosum) detected by inter-simple sequence repeats (ISSRs). Genome 45: 481-484.

Chowdhury M.A., Vandenberg B. and Warkentin $T$ (2002). Cultivar identification and genetic relationship among selected breeding lines and cultivars in chickpea (Cicer arietinum L.). Euphytica 127:317-325.

Eleuch L., Jalil A., Grando S., Ceccarelli S., Schmising M.K., Tsujimoto A., Daaloul A. and Baum M. (2008). Genetic Diversity and association analysis for salinity tolerance, heading date and plant height of barley germplasm using simple sequence repeat markers. J. Integr. Plant Biolo. 50(8):10051015.

Fernández ME., Figueiras AM. and Benito C. (2002). The use of ISSR and RAPD markers for detecting DNA polymorphism, genotype identification and genetic diversity among barley cultivars with known origin. Theoretical and Applied Genetics 104: 845851 .

Joshi S.P., Gupta V.S., Aggarwal R.K., Ranjekar, P.K. and Brar, D.S. (2000). Genetic diversity and phylogenetic relationship as revealed by inter-simple sequence repeat (ISSR) polymorphism in the genus Oryza. Theoretical and Applied Genetics 100:1311-1320.

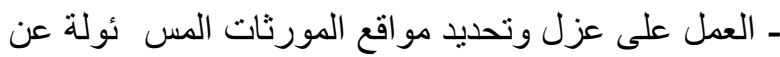

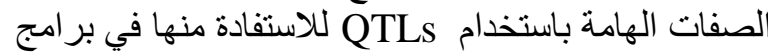
التربية واستخدامها كآباء في عمليات التهجين.

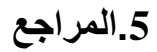

سيد، محمود هيثم.( المع 2001).استخدام مؤشرات من الدنا DNA

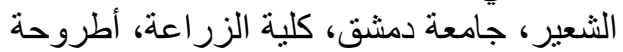

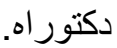

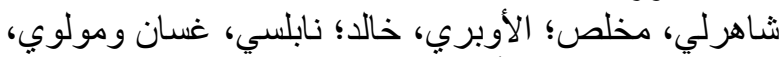

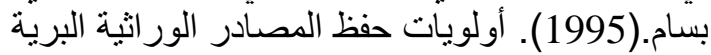

$$
\text { في سوريا، دمشت، سوريا. }
$$

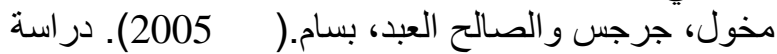

Fabaceae فصنيفية لجنس.Medicago L (فيلة

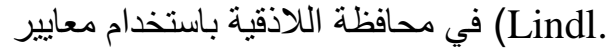
مورفولوجية وتشريحية، مجلة جامعة تشرين

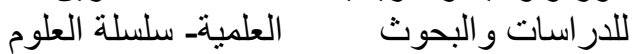

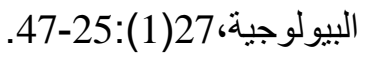

\section{REFERENCES}

Anand A., Baig M.J. and Mandal, P.K.(2000). Response of alfalfa genotypes to saline water irrigation. Biol. Plant. 43: 455-457.

Ayad W.G., Hodgkin T., Jaradat A and Rao V.R.(1997). Molecular genetic techniques for plant genetic resources. Report for an IPGRI workshop, IPGRI, Rome, Italy, p.p. 11-12.

Bauchan G.T. and Green S. (2000). Report on the Status of Medicago Germplasm in the United States. Alfalfa C. G. C., USDA-ARS. 
Karp A., Kresovich S., Bhat K. V., Ayad W. G. and Hodgkin, T.(1997). Molecular tools in plant genetic resources conservation: a guide to the technologies. 1st ed. IPGRI Technical Bulletin NO. 2. IPGRI, Rome, Italy, pp. 9-21.

Kijas J.M.H., Fowler J.C.S. and Thomas M.R.(1995). An evaluation of sequence tagged microsatellite site markers for genetic analysis within Citrus and related species. Genome 38:349-355.

Lane A. (2007). An introduction to crop wild relatives, Geneflow, Publication about Agricultural Biodiversity, Biodiversity International, $\mathrm{p}: 19$.

Maniatis T., Fritsch E.F and Sambrook J. (1982). Molecular cloning: Laboratory Manual. Cold Spring Harbor Laboratory Press, Cold Spring Harbor/ NY.

Nagaoka T. and Ogihara Y.(1997). Applicability of inter-simple sequence repeat polymorphisms in wheat for use as DNA markers in comparison to RFLP and RAPD markers. Theoretical and Applied Genetics 94: 597-602.

Nei M. (1987). Molecular Evolutionary Genetics. Columbia University Press, New York.

Petolescu C. and Nedelea G.(2009). Genetic Diversity Analysis of the In Vitro Regenerated Alfalfa Plants Using Inter Simple Sequence Repeat (ISSR) Markers. Romanian Biotechnological Letters ,1416: 4882-4886.

Powell W., Morgante M., Doyle J.J., Mcnical J., Tingey S.V. and Rafalski A.J. (1996). Genepool Variation in Genus Glycine Subgenus Soja Revealed by polymorphic Nuclear and chloroplast microsatellites, Genetics 144:793-803.

Qi X., Stam P. and Lindhout P.(1996). Comparison and integration of four barley genetic maps. Genome 39:379-394.

Rafalski, J.A.,Vogel J.M., Morgante M., Powell W., Andre C and Tingey, S.V.(1996). Generating and using DNA markers in plants. No mammalian Genomic Analysis: A Practical Guide. 4:75-134.

Ramsay L., Macaulay M., Degli Ivanissevich S.,
Maclean K., Carsle L., Fuller J., Edwards K.J., Tuvesson S., Morgante M., Massari A., Maestri E., Marmiroli N., Sjakste T., Ganal M., Powell W. and Waugh R. (2000). A simple sequence repeat- based linkage map of barley. Genetics 156:1997-2005.

Saiki R.K., Gelfand, D.H., Stoffel S., Scharf S.J., Higuchi R., Horn G.T., Mullisand K.B. and Eriich H.A.(1988). Prime-directed enzymatic amplification of DNA with a thermostable DNA polymerase. Science 239:487-491.

Saiki R. K., Scharf S., Faloona F., Mullis K. B., Horn G. T., Eriich H. A. and Amheim,N. (1985). Enzymatic amplification of b-globulin genomic sequences and restriction site analysis for diagnosis of sickle cell anaemia. Science 230:1350-1354.

Sigovia- Lerma, A., Cantrell, R. G., Conway, J. M. and Ray, I. M.(2003). AFLP- based assessment of genetic diversity among nine alfalfa germplasms using bulk DNA templates. Genome 46: 51-58.

Touil L., Guesmi F., Fares K., Zagrouba, C. and Ferchichi A. (2008). Genetic Diversity of Some Medditerranean Populations of the Cultivated Alfalfa (Medicago stiva L.) Using ISSR Markers, Pakistan Journal of Biological Sciences11(15): 1923-1929.

Tragoonrung S., Kanazin V., Hayes P.M. and Blake T.K. (1992). Sequence tagged site facilitated PCR for barley genome mapping. Theor. Appl. Genet. 84:1002-1008.

Valizadeh M., Kang K. K., Kanno A and Kameya, T.(1996). Analysis of genetic distance among nine Medicago species by using DNA polymorphisms. Breeding Science 46:pp. 7-10

Williams J.G.K., Kubelik A.R., Livak K.J., Rafalski J.A and Tingey S.V. (1990). DNA polymorphisms amplified by arbitrary primers are useful as genetic markers. Nucleic Acids Research 18(22) 6531-6535.

Ziekiewicz E., Rafalski A. and Labuda A (1994). Genome fingerprinting by simple sequence repeat (SSR) anchored polymerase chain reaction amplification. Genomics 20:178-183. 